\title{
Development of an Optimal Piezoelectric Transducer to Excite Guided Waves in a
}

\section{Rail Web}

\author{
Dineo A. Ramatlo ${ }^{a *}$, Daniel N. Wilke ${ }^{b}$ and Philip W. Loveday \\ ${ }^{\mathrm{a}, \mathrm{b}}$ University of Pretoria, ${ }^{\mathrm{a}, \mathrm{c}}$ CSIR Materials Science and Manufacturing; South Africa

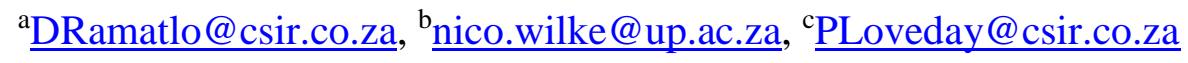

\begin{abstract}
Ultrasonic transducers have demonstrated the ability to effectively excite guided waves that can propagate over long distances and are thus employed in monitoring systems. An ultrasonic piezoelectric transducer that strongly excites a mode travelling in the web of the rail is required to detect cracks in the rail web. A numerical method referred to as the Coupled Semi-Analytical Finite Element - 3 Dimensional Finite Element (Coupled SAFE-3DFE) method is employed to model the excitation of guided waves in a rail by a transducer. The aim of this study is to demonstrate that the Coupled SAFE-3DFE method used in conjunction with mathematical optimization methods, can automate the optimal design of a transducer. The optimal transducer was constructed and experimental measurements were found to agree with the predicted performance.
\end{abstract}

\section{Keywords}

Guided Waves, Coupled SAFE-3DFE, Piezoelectric Transducer, Optimization, Response Surface 


\section{Introduction}

Guided Wave Ultrasound has demonstrated the capability to detect defects in rail tracks [1]. One advantage of this method is that it is possible to inspect and monitor structures over long ranges. It has been shown that a mode propagating mainly in the head of the rail (referred to as the head mode in this paper) can be used to detect defects in the rail head at long distances [2]. In order to detect defects in the web of the rail it is necessary to use a propagating mode with energy concentrated in the web section of the rail. One such mode, referred to as the web mode in this paper was identified by Long and Loveday [3]. They demonstrated that the mode is suitable for detecting welds and damage in the web of the rail. The dispersion curves and illustrations of the head and web modes of a UIC60 rail are shown in Figure 1.

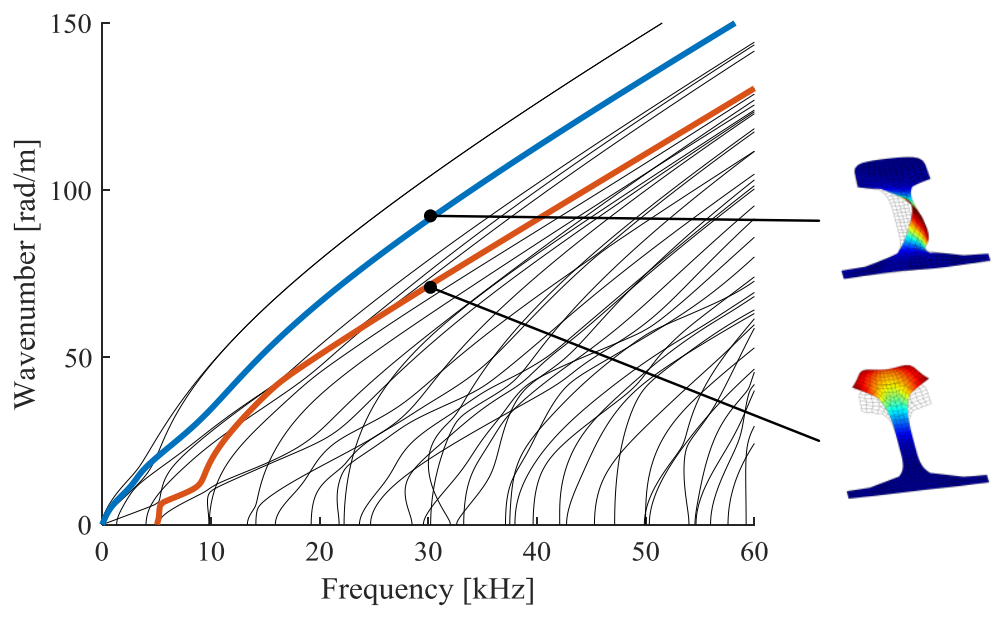

(a)

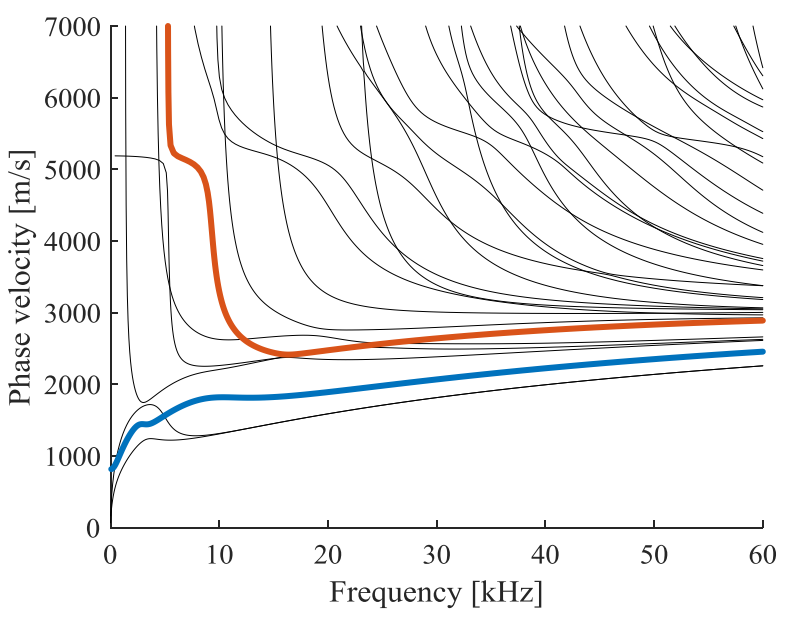

(b)

Figure 1. (a) Wavenumber and (b) phase velocity dispersion curves of UIC60 Rail and mode shapes of the web and head modes at $35 \mathrm{kHz}$.

As a transducer suitable for exciting the head mode has been developed, there is now a requirement to develop an ultrasonic piezoelectric transducer to effectively and optimally 
excite the web mode at a specified frequency. Instead of following the conventional trial and error approach that requires multiple transducers to be manufactured and tested, a numerical modelling based design approach outlined in this paper is followed where only the final design is manufactured to validate the performance. The ability of a transducer to effectively excite guided waves in a rail track can be influenced by many factors, with material and geometrical properties being the most important. To achieve the set design goals, these factors need to be carefully selected, which we demonstrate to be possible if a systematic design approach is followed. In this paper, the design process is simplified as material properties of the transducer and the geometry of the piezoelectric stack are kept constant. This decision was made to allow the use of the same piezoelectric stack and manufacturing techniques that are used in the transducer for the head mode, which is already in production. The design problem in this paper is thus to find the optimal sizes of the back and front masses of the web transducer.

First, an analysis approach is required that can reliably predict the propagating waves excited by a transducer attached to a rail track. A number of techniques have been demonstrated to be reliable, including the computationally demanding full threedimensional (3D) finite element analysis of the rail track and the transducer. The computational demand of full 3D analyses of the rail track often prohibits numerous analyses to be conducted. An alternative technique and computationally efficient approach is the previously developed Coupled SAFE-3DFE method [4,5], which combines the Semi-Analytical Finite Element (SAFE) model of the rail and a 3D finite element model of the transducer.

Adopting a technique referred to as the SAFE method, we can perform the numerical modelling of ultrasonic guided waves in a rail track. Researchers in [6] and [7] have 
highlighted the reliability and efficiency of the SAFE method; hence the method has recently become widely adopted for studying wave propagation in rails. In the SAFE method, only a 2D cross-sectional mesh of a waveguide is employed in the computations, while the length of the waveguide is treated analytically. The method is therefore attractive due to its improved computational efficiency compared to 3D finite element methods. The problem size is not dependent on the length of the waveguide, but rather on the discretization of only the cross-section. The computational demand in the SAFE method is concentrated in the computation of the eigenvalues and corresponding eigenvectors (mode shapes) to describe the different modes of propagation that a waveguide supports. This eigenvalue problem is computationally demanding but fortunately it only has to be solved once for the rail if the eigenvalues and eigenvectors are stored and used during the analysis of numerous transducer designs attached to the rail.

A Coupled SAFE-3DFE method for modelling the excitation of guided waves by a piezoelectric transducer was proposed by Loveday [4,5]. The method employs a 3D finite element mesh of the transducer and attaches it to a 2D SAFE mesh of the waveguide through coincident finite element nodes. An improved version of the method, which allows for the attachment of the transducer to the waveguide model through noncoincident nodes was later proposed by Loveday and colleagues at the CSIR. Ramatlo et al. [8] demonstrated that the Coupled SAFE-3DFE method accurately predicts the excitation of guided wave modes by a piezoelectric transducer. The modelling of the transducer in $3 \mathrm{D}$ is necessary as the interest is on designing resonant transducers to achieve powerful excitation and long-range propagation of guided waves. An appropriate 3D transducer model ensures that the dynamics of the transducer are correctly captured. 
In this study the Coupled SAFE-3DFE method is used to predict the capability of a transducer to excite guided waves in the rail. Again, the computational efficiency of the Coupled SAFE-3DFE is evident as only the transducer changes, while the rail model remains the same allowing us to store the eigenvalues and mode shapes of the waveguide.

Searching for the optimal transducer design can become inefficient when a trial and error approach is followed. However, if Coupled SAFE-3DFE is used in conjunction with appropriate optimization methods, the design process can be practical and computationally acceptable. Arora [9] outlines the typical steps of a design optimization procedure. Firstly, the variables that dictate the design and are allowed to change need to be identified and are referred to as design variables. For the problem under consideration in this study, the design variables parameterize parts of the geometry of the transducer. The performance of each design needs to be quantified by a single scalar value referred to as the objective function. The set of designs that are valid is limited by constraints imposed on the design variables and reflects the feasible design domain.

Design optimization is generally an iterative process and primarily requires the evaluation of many designs until no improvement to the design can be made. In our problem the objective function could be potentially noisy due to re-meshing between transducer designs. We therefore opt for a response surface based method as opposed to a direct gradient based approach to smooth out the response between designs. In the response surface method, we first conduct a Design of Experiments (DOE) where we select a finite set of transducer designs randomly scattered within the design space. Their performances are then simulated using Coupled SAFE-3DFE. This approach allows us to compute multiple designs concurrently (depending on the number of available computers and CPUs) whereas a direct gradient-based approach is inherently sequential. 
Given a finite set of randomly scattered transducer designs and corresponding transducer performances (objective function values), we approximate the objective function by a response surface that is an accurate representation of the simulated performances from the Coupled SAFE-3DFE model over the entire feasible design space. We can then interpolate on the response surface to predict the performance of other transducer designs which have not been analysed. In this paper, the design space is sampled using a Latin Hypercube sampling strategy (to provide a number of designs to be analysed) and the response surface is constructed using a Radial Basis Function (RBF) interpolation scheme. The response surface is smooth by construction and computationally efficient to evaluate.

The optimal transducer design can then be found by maximizing the response surface model using a conventional optimization algorithm (we choose the Nelder-Mead algorithm). The process steps to obtain an optimal transducer design using the response surface method is summarized in Figure 2.

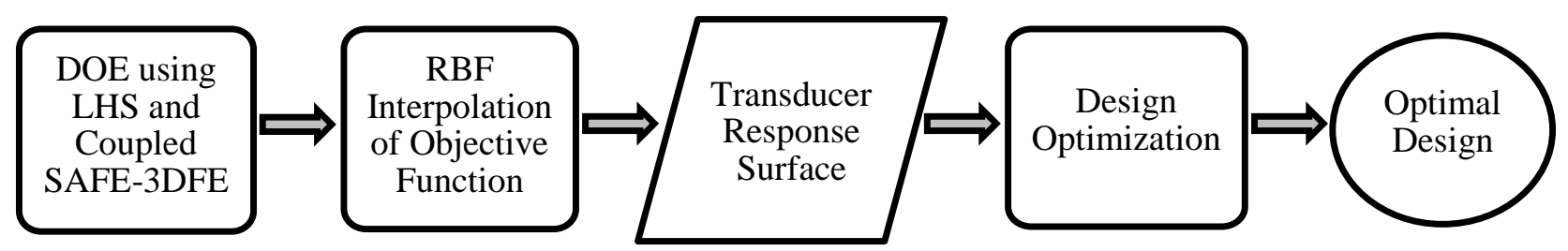

Figure 2. Process steps to obtain an optimal transducer design using the response surface method.

Given a specific waveguide model, the advantages of using Coupled SAFE-3DFE with response surface based optimization methods are as follows: 
- The computational demand of Coupled SAFE-3DFE is an eigenvalue problem that needs to be solved at a set of frequencies.

- Multiple transducer designs can be evaluated concurrently on different computers or different CPUs.

- Finding the optimal transducer is automated; implying that once the optimization problem is set-up, an automated process is followed until the optimal transducer is found.

- The designer's time is only required to interpret the optimal solution or to modify the optimization problem.

The main objective of this paper is to demonstrate a procedure for designing optimal transducers for guided wave excitation in rails by adopting the Coupled SAFE-3DFE method, the response surface method and the Nelder-Mead optimization algorithm. The Coupled SAFE-3DFE method is outlined in Section 2 of the paper. The response surface method and the optimization process are described in Section 3 while the results and discussion of results are presented in Section 4. The conclusions are drawn in Section 5 where the recommendations for future work are stated.

\section{Coupled SAFE-3DFE Model}

The SAFE method has recently become widely adopted by researchers. Only a brief description of the SAFE method is included here. A thorough explanation of the method can be found in references $[6,7]$.

The governing equation of motion for a SAFE model is given by: 


$$
\begin{array}{cc}
{\left[\begin{array}{cc}
K_{0}-\omega^{2} M & 0 \\
0 & -K_{2}
\end{array}\right]\left\{\begin{array}{c}
U \\
\kappa U
\end{array}\right\}+\kappa\left[\begin{array}{cc}
-j K_{1} & K_{2} \\
K_{2} & 0
\end{array}\right]\left\{\begin{array}{c}
U \\
\kappa U
\end{array}\right\}=\left\{\begin{array}{l}
F \\
0
\end{array}\right\},} \\
\text { or } \\
\left.[A-\kappa B]\} \begin{array}{c}
U \\
\kappa U
\end{array}\right\}=\left\{\begin{array}{l}
F \\
0
\end{array}\right\}
\end{array}
$$

where $K_{0}, K_{1}$ and $K_{2}$ are the system stiffness matrices, $M$ is the mass matrix, $\omega$ is the natural frequency of the system and $\kappa$ is the wavenumber. $F$ is the force applied to the waveguide and $U$ is the displacement defined as in the formulation presented by Gavric [7].

The response of a waveguide at a distance $z$ from the excitation point is given by:

$$
U(z)=\sum_{r=1}^{3 N} \alpha_{r} \psi_{r}
$$

where $\psi_{r}$ is the eigenvector of mode $r$ (found from equation 1 with $F=0$ ), and the term $\alpha_{r}$ is the modal amplitude evaluated as:

$$
\alpha_{r}(z)=-j \frac{\psi_{r}^{T} F}{\psi_{r}^{T} B \psi_{r}} e^{-j \kappa_{r} z}
$$

In the Coupled SAFE-3DFE method, a 3D finite element model of an electrically driven transducer is attached to the 2D SAFE model of the rail to excite the mode of interest. A schematic representation of the Coupled SAFE-3DFE method is illustrated in Figure 3. A 2D cross-section of the infinite rail is extracted and modelled using four node quadrilateral elements in SAFE. To excite ultrasonic guided waves in the web of the rail, the $3 \mathrm{D}$ transducer model is attached to the edge of the web cross-section, at the centre where it will excite the waveguide to propagate the web mode. The transducer model is driven by a voltage signal generated from a 17.5 cycle hanning-windowed tone burst with a centre frequency of $35 \mathrm{kHz}$. For effective modelling of the problem, model data will 
need to be accurately mapped between the 2D SAFE model and the 3D transducer model. This is explained in Loveday [5] and Ramatlo et al. [8].

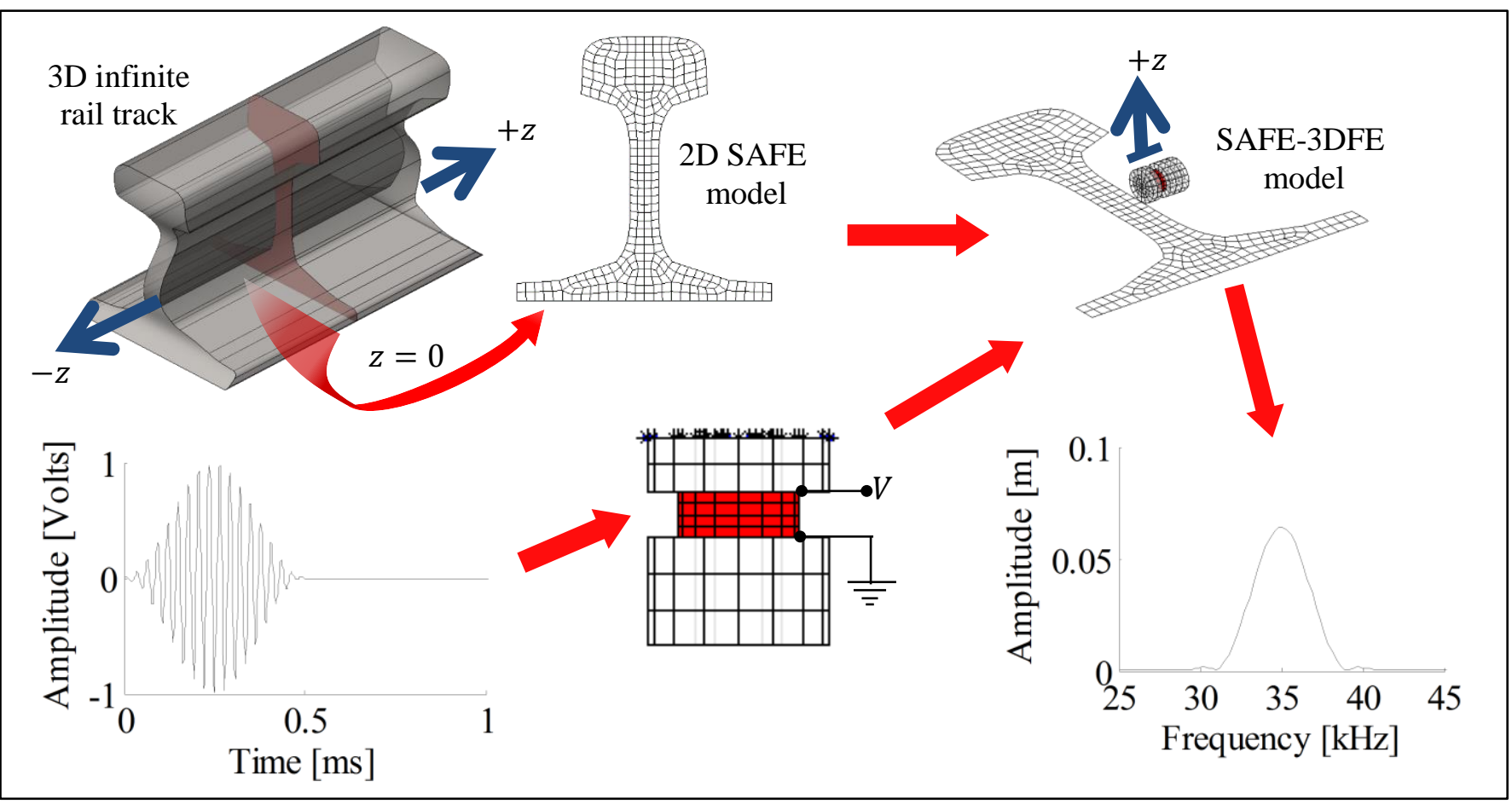

Figure 3. Schematic representation of the Coupled SAFE-3DFE method.

Firstly, for the 2D SAFE model, the response of the rail to applied unit loads is computed using equations 2 and 3. Following the formulation in Loveday [5] and Ramatlo et al. [8], the dynamic stiffness of the rail experienced by the transducer can be estimated from the known applied loads $\left(F_{\text {in }}\right)$ and the computed responses $U_{i n}$, at the interface degrees of freedom (DOFs):

$$
D_{r} U_{i n}=F_{i n}
$$

The 3D finite element model of the transducer includes piezoelectric elements [10] and the equations of motion have the form: 


$$
M \ddot{u}+\left[\begin{array}{cc}
K_{u u} & K_{u \phi} \\
K_{u \phi}^{T} & K_{\phi \phi}
\end{array}\right]\left\{\begin{array}{l}
u \\
\phi
\end{array}\right\}=\left\{\begin{array}{l}
f \\
q
\end{array}\right\}
$$

where $q$ is the electrical charge and $\phi$ is the electric potential.

When the transducer is excited, voltages are applied to the flat surfaces of each piezoelectric ring, via the silver electrodes. In our model of the transducer each piezoelectric ring is modelled by only one linear finite element through the thickness of the ring. Therefore all voltage degrees of freedom in the model are on an electrode. There are no voltage degrees of freedom that are not specified. Due to this simplification the harmonic displacement response may be written from the first equation in equation 5 as:

$$
\left[K_{u u}-\omega^{2} M\right] u=-K_{u \phi} \phi_{s}+f
$$

where $\phi_{s}$ is a vector of specified voltages applied at the electrical potential DOFs. From equation 6 , we see that the electrical voltage excitation multiplied by the piezoelectric coupling matrix acts similarly to an externally applied force. We can write this term as an electrical force:

$$
f_{v}=-K_{u \phi} \phi_{s}
$$

The term on the left hand side of equation 6 is the dynamic stiffness of the transducer. The dynamic stiffness of the transducer $D_{t}=\left[K_{u u}-\omega^{2} M\right]$ can be partitioned into DOFs in contact with the rail (in) and DOFs not in contact $(n)$. The boundary condition imposed by the presence of the rail may be included in the transducer model by adding the dynamic stiffness of the rail to the dynamic stiffness of the transducer at the interface DOFs.

$$
\left[\begin{array}{cc}
D_{t \text { nn }} & D_{t \text { nin }} \\
D_{t \text { inn }} & D_{t \text { inin }}+D_{r}
\end{array}\right]\left\{\begin{array}{l}
u_{n} \\
u_{i n}
\end{array}\right\}=\left\{\begin{array}{l}
f_{v n} \\
f_{v \text { in }}
\end{array}\right\}
$$


Equation 8 is solved at each frequency to obtain the transducer displacements. The displacements at the interface DOFs $u_{i n}$, are then used to determine the forces applied to the rail using equation 4. Finally, these forces are applied to the SAFE model of the rail and the response of the rail is solved at each frequency using equations 2 and 3.

\section{Optimal Design of a Transducer}

The goal is to obtain an optimal transducer design for exciting the web mode. The design is of a sandwich type with the structural configuration shown in Figures $4 \mathrm{a}$ and $4 \mathrm{~b}$. The piezoelectric transducer will be driven by a $35 \mathrm{kHz}$ centre frequency hanning-windowed tone burst signal with 17.5 cycles. Such a signal contains energy in the frequency range we plan to use in our system. The amount of energy that the transducer can transmit to the rail web mode must be maximized.

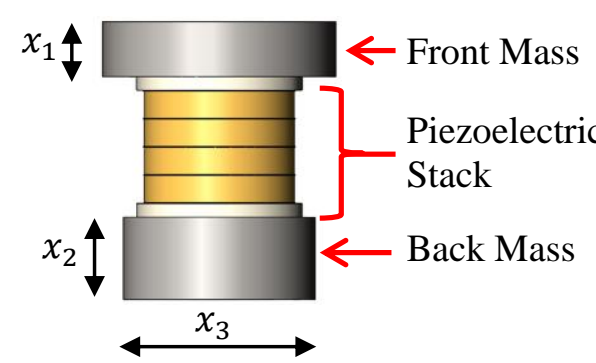

(a)

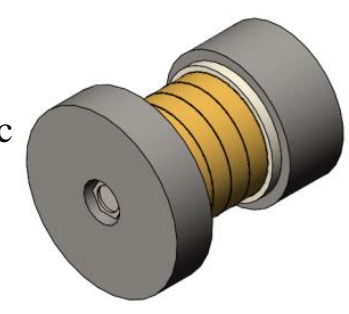

(b)

Figure 4. The (a) side and (b) isometric views of the transducer showing its structural composition.

The front and back masses of the transducer are made of steel while the piezoelectric material is a Type IV PZT. The aim of the design problem is to determine the front mass and back mass dimensions. The geometry and material of the piezoelectric stack is kept constant as explained in the introduction. To allow for the use of an available transducer 
housing, the diameter of the front mass is kept constant at a size compatible with the housing. The design problem therefore contains the three design variables $x_{1}, x_{2}$ and $x_{3}$ shown in Figure 4a.

Although fixing the diameter of the front mass poses a limitation on the design problem, it provides notable advantages, which include reduced problem complexity and improved computational cost and time.

Due to construction requirements, the thickness of both the front and back masses are limited to be between $4 \mathrm{~mm}$ and $10 \mathrm{~mm}$, while the back mass diameter is limited to be between $12.05 \mathrm{~mm}$ and $15.5 \mathrm{~mm}$.

\subsection{Mathematical Design Optimization Model}

Following the discussion of the design of the rail web transducer above, the standard mathematical design optimization problem is stated as follows:

Find a vector $\boldsymbol{x}=\left\{x_{1}, x_{2}, x_{3}\right\}^{T}$ containing the design variables: front mass thickness $x_{1}$, back mass thickness $x_{2}$ and back mass diameter $x_{3}$, to maximize the response of the rail web mode to piezoelectric transducer excitation given by some relevant objective function $f(\boldsymbol{x})$, that is continuous and is subject to three inequality constraints:

$$
\begin{gathered}
4 \leq x_{1} \leq 10 \\
4 \leq x_{2} \leq 10 \\
12.05 \leq x_{3} \leq 15.5
\end{gathered}
$$

\subsection{Objective Function}


We have defined the excitation of the transducer and we want to obtain the maximum response to this excitation signal. The SAFE-3DFE model can predict the modal amplitude, frequency response and time history of guided waves excited by a particular transducer design. The modal amplitude captures the dynamics of the transducer when attached to the rail. Consider the predicted modal amplitudes of the web mode for three candidate transducer designs denoted A, B and C, plotted in Figure 5a. It is seen from Figure 5a that the modal amplitude can have abrupt changes in amplitude at certain frequencies. It is believed that this might be due to the effect of cut-on frequencies of other modes. Therefore, although the modal amplitude captures the dynamics of the transducer, it is not advised to be used as an optimization criterion directly as it is not a smooth function.

Alternatively, consider the response of the web mode when the excitation signal is applied to the three candidate transducer designs, plotted in Figure 5b. This function is obtained by multiplying the modal amplitude by the spectrum of the excitation signal and the mode shape of the web mode. The time history of the web mode can be computed by taking the inverse Fast Fourier transform of the response function and is shown in Figure 5c. 


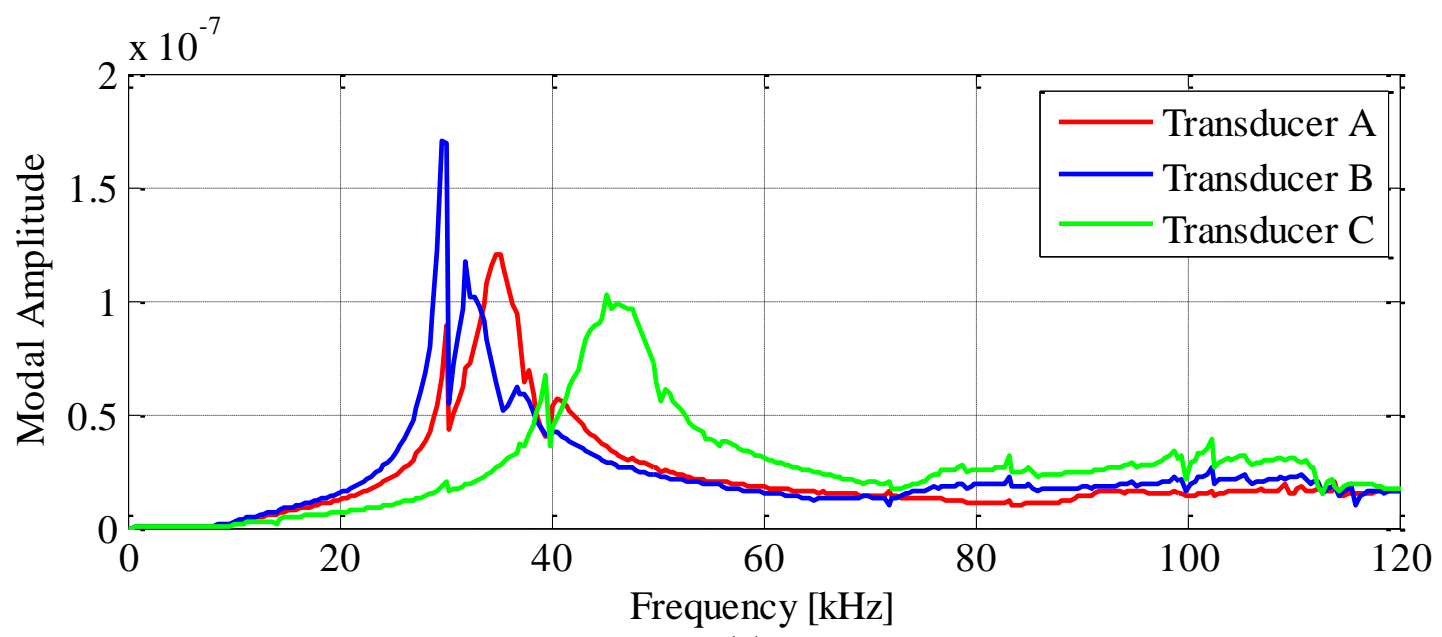

(a)

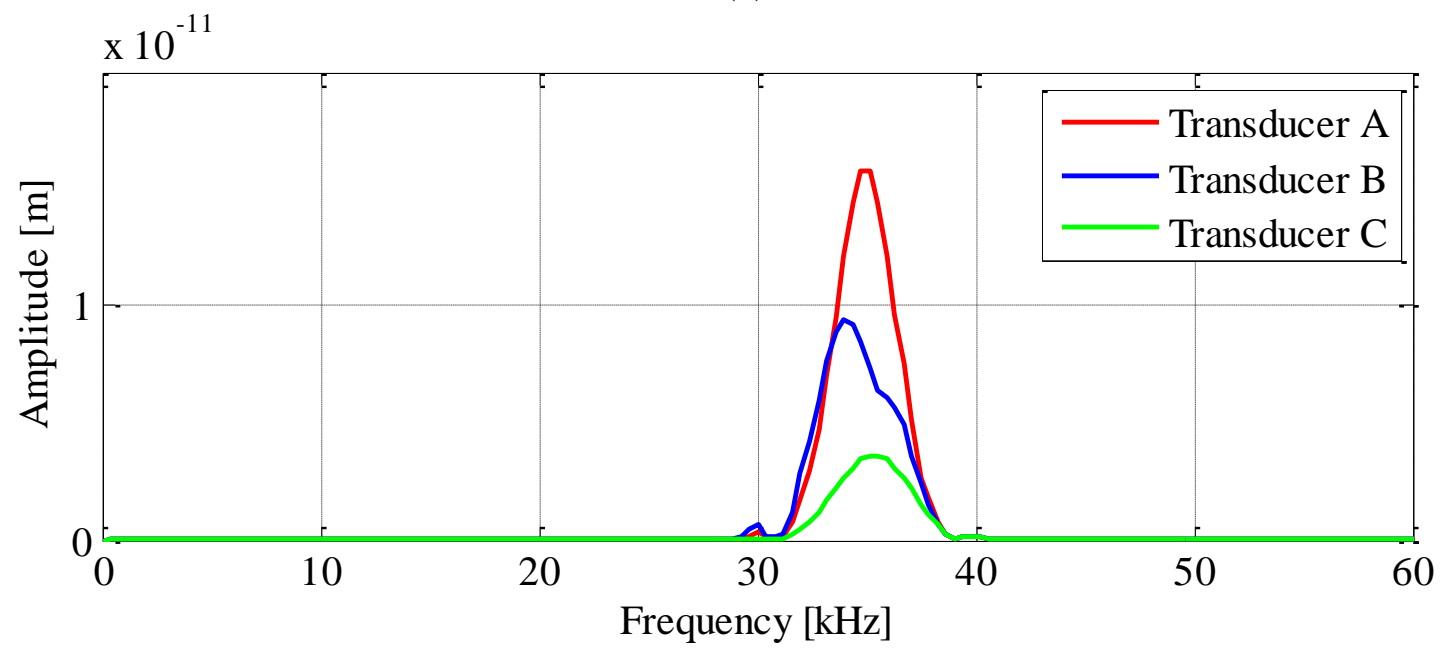

(b)

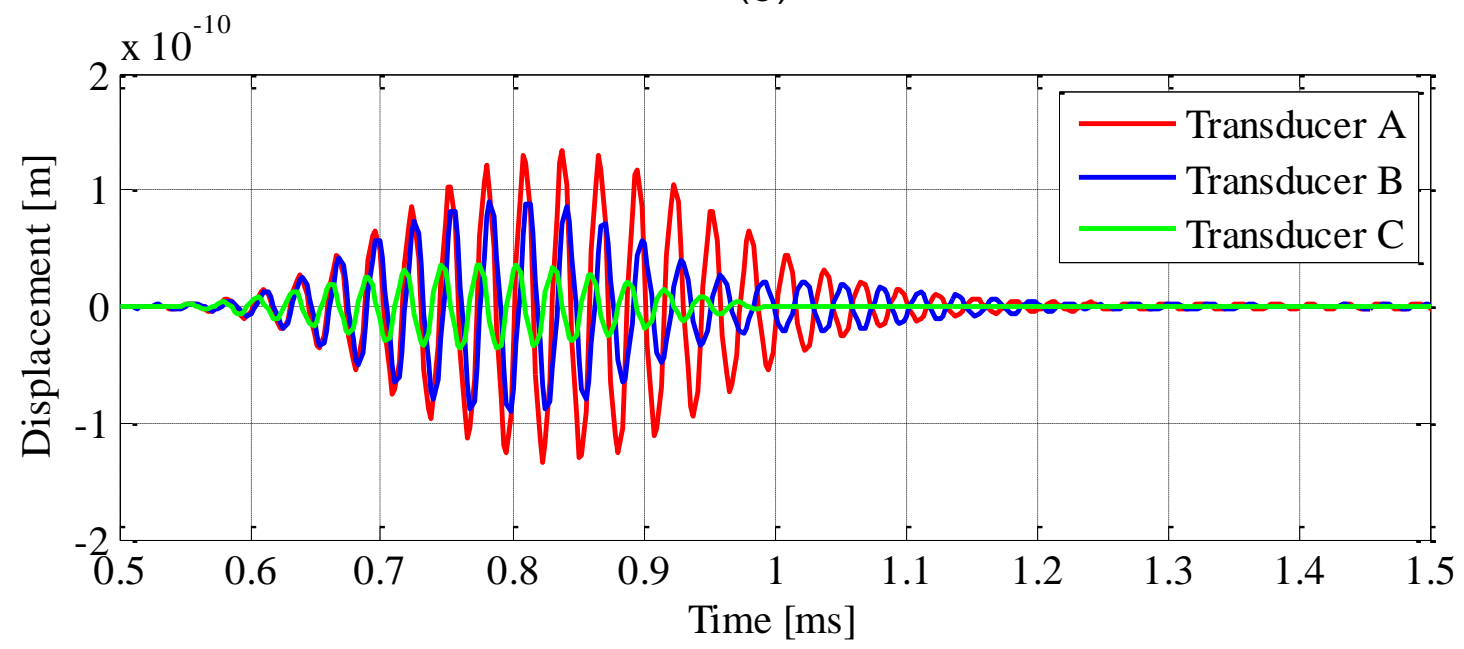

(c)

Figure 5. The (a) modal amplitude, (b) frequency response and (c) time responses of the web mode when excited by different transducer models. 
The objective of the design is to transfer the maximum energy possible into the web mode. If the web mode is strongly excited by a transducer, the peak amplitudes of the time history and the frequency response function will be large. An optimization criterion must be represented by a single value that will indicate whether a design is good or not depending on the objective statement. It would be possible to use the value of the response at $35 \mathrm{kHz}$ as an objective function. However, it is possible that abrupt changes in this response could occur and we therefore choose to use the area under the frequency response function. In Figure $5 \mathrm{~b}$, the objective function value for each transducer design would be the area below their specific frequency response curve. The area below each curve is computed by taking the integral of the curve between the limits of $0 \mathrm{~Hz}$ and $120 \mathrm{kHz}$. The area of the frequency response function provides a smooth and continuous function for the optimization criterion.

\subsection{Response Surface Construction}

Recall the process outlined in Figure 2, instead of optimizing the problem directly by evaluating the response from the Coupled SAFE-3DFE for each design, we first approximate the objective function using a response surface. This is done by first evaluating a finite set of designs using Coupled SAFE-3DFE to get their responses. The response surface method is then used to interpolate the objective function values of the other designs that were not explicitly evaluated. The approximation of the objective function is constructed using RBF interpolation. The benefit of this approach is that the designs can be evaluated in parallel when approximating a response surface, whereas, 
directly optimizing the problem requires sequential analyses. In addition, a model of the response is available for further interrogation that is computationally efficient to evaluate.

The first step in constructing a response surface is to sample the design space. One way to select a set of random transducer designs that are evenly distributed over the design space is to use the Latin Hypercube sampling (LHS) method $[11,12]$. The LHS method involves a full stratification of the sampled distribution, with a random selection inside each stratum. To sample an s-dimensional space with $N$ data points, the sampling space is divided into a series of strata by dividing the range of each variable into $N$ equally sized intervals. The $N$ sampling points are then randomly distributed over the sampling frame such that each stratum contains only one data point, which enforces coverage of the domain for the chosen number of points.

To illustrate the process we first demonstrate it on a simplified 2-parameter problem. An example of a 2-dimensional space sampled with 5 data points using LHS is illustrated in Figure 6. The variables are treated independently and each row/column is randomly sampled with one point only.

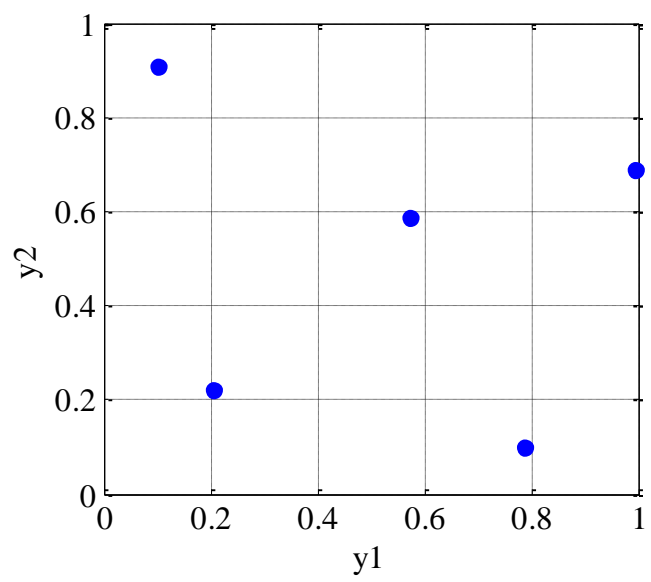


Figure 6. Illustration of a simplified 2-dimensional space sampled with $N=5$ LHS points.

The LHS generated designs are then analysed explicitly to compute their function values. For this example problem we can assume that we are approximating a planer surface, so each LHS design is given a weighting of one as the objective function value. Once this is done, we are able to construct a response surface to approximate the actual objective function which is a planer surface for this problem. Using RBF interpolation, the objective function $f(x)$ is approximated by a linear combination of non-linear basis functions [13]:

$$
f(x)=\sum_{j=1}^{N} c_{j} \varphi\left(\left\|x-x_{j}\right\|\right)
$$

where $c_{j}$ are the weights by which the basis function is scaled. The basis functions are radially symmetric about their respective centre points $x_{j}$ which are the sampled LHS points. The weights $c_{j}$ are solved from a linear system that ensures that the responses at the LHS designs are exactly recovered. In this paper, an RBF approach using a Gaussian basis function

$$
\varphi(x)=e^{-(\epsilon\|x\|)^{2}}
$$

is adopted. Here, $\epsilon$ is a positive real number called the shape parameter that affects the accuracy and numerical stability of the response surface. A good value of the RBF shape parameter $\epsilon$, can be computed by minimizing the leave-one-out cross validation (LOOCV) error [14]. Figure 7 illustrates the approximated response surface for the DOE data in Figure 6. This is a poor approximation of a planer surface, and to improve it more LHS points should be used. 


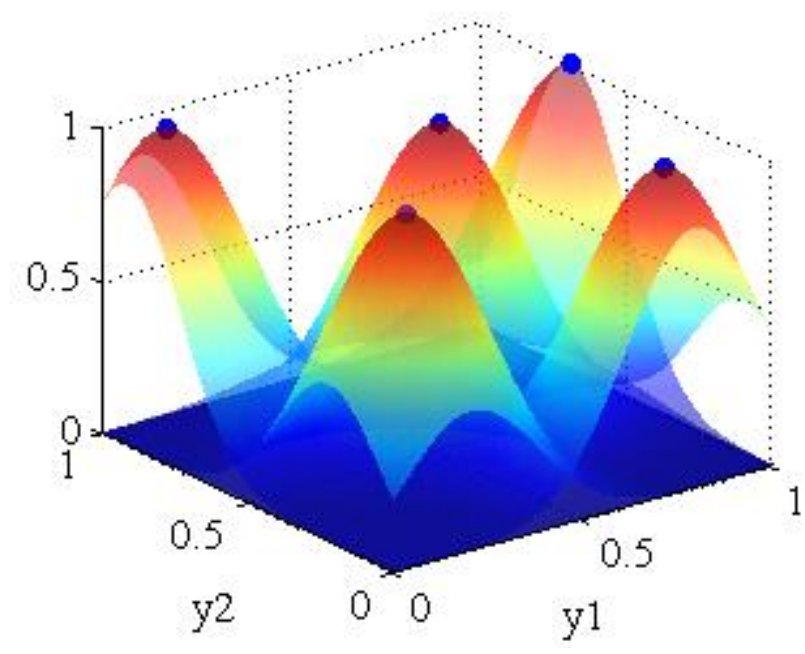

(a)

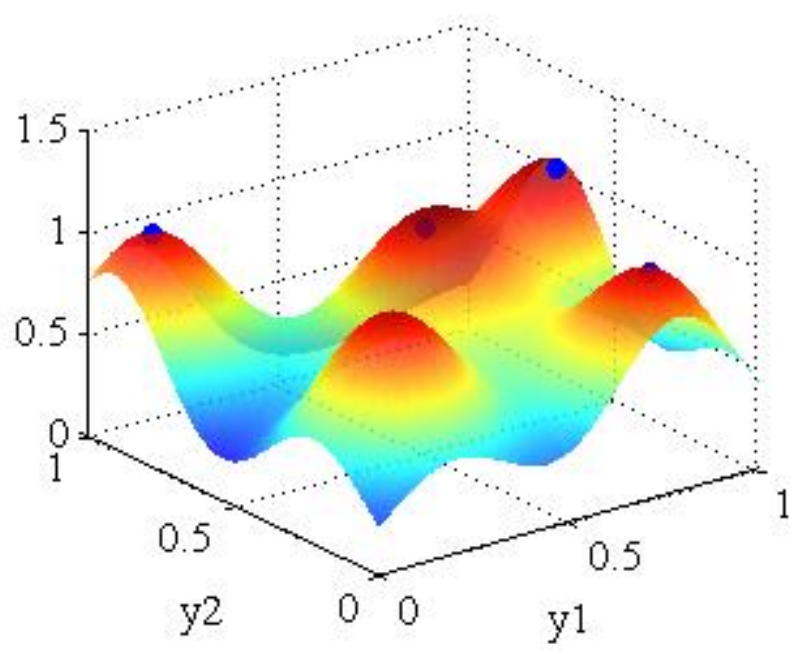

(b)

Figure 7. Illustration of (a) Basis functions and (b) Response surface construction using $\mathrm{RBF}$ for the simplified 2-parameter problem.

In RBF interpolation, the response surface usually has larger errors at and close to the edges of the sampled space. Although this is well known, it is seldom reported or considered when constructing the DOE. The DOE is usually constructed only within the feasible design domain, resulting in poor approximations close to the edges of the design domain. In this study we deliberately construct the DOE larger than the feasible design domain to avoid inaccuracies close to the boundaries as depicted in Figure 8. To demonstrate this we locate the feasible design domain (indicated as "Design Space" in Figure 8) within the DOE sampled domain (indicated as "Sampling Space" in Figure 8) non-symmetrically for this study only, to avoid difficulties when meshing the transducer geometry. If meshing was not a problem, we would have the feasible design domain located centrally within the DOE sampled space. The sampling space was sampled with 125,250 and 500 data points respectively, to evaluate the effect of sample size. 


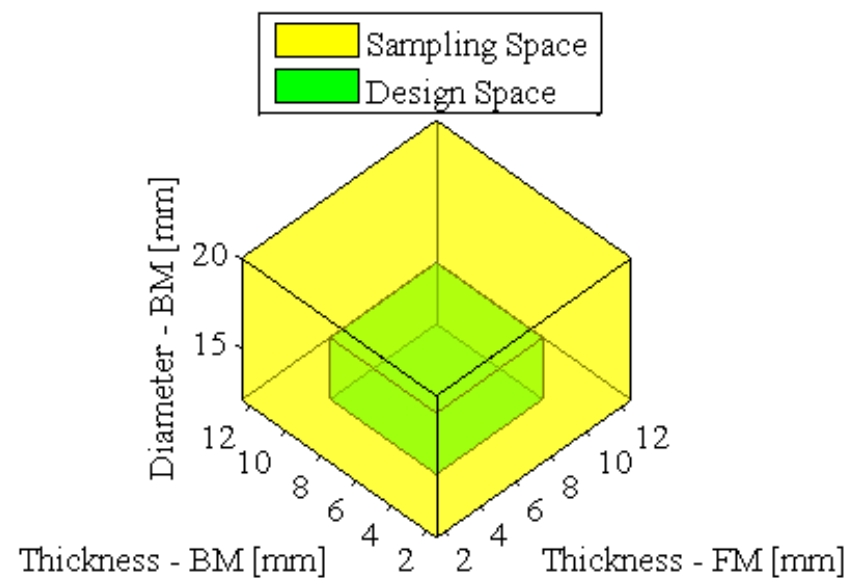

Figure 8. The sampling space and design space, with the sampling space chosen deliberately larger than the design space.

Once the response surface had been approximated, the derivative-free Nelder-Mead minimizer [15] was used to find the optimal design. This optimizer is implemented in fminsearch in Matlab and is designed to minimize multidimensional unconstrained non-linear minimization problems. The constrained optimization problem presented is transformed to an unconstrained problem by using the exterior penalty method [9]. The exterior penalty penalises a design when a constraint is violated by adding a positive value proportional to the violation to the objective function, driving the minimizer back to the feasible domain. The constrained maximization problem is formulated as an unconstrained minimization problem for the Nelder-Mead minimizer:

Find a vector $x=\left\{x_{1} ; x_{2} ; x_{3}\right\}$ of design variables that minimizes:

$$
\Phi(\boldsymbol{x})=-f(\boldsymbol{x})+p_{1} \sum_{x_{i}<L B}\left(x_{i}-L B_{i}\right)^{2}+p_{2} \sum_{x_{i}>U B}\left(x_{i}-U B_{i}\right)^{2}
$$

The upper and lower bound penalty parameters $p_{1}$ and $p_{2}$, were selected as $5 \times 10^{7}$. 
To avoid confusion and aid the discussions that follow, this unconstrained minimization problem is only to aid the Nelder-Mead minimizer, the discussions that follow will always refer the original maximization problem and bound constraints.

\section{Results}

\subsection{Design Optimization}

The three sets of DOEs and their associated objective function values computed using the Coupled SAFE-3DFE method are plotted in Figures 9a-9c. One attractive feature of DOE is that it provides the designer with informative output data, in particular when the number of design variables is limited to a few. It is clear from Figures 9a-9c that the data gives a good indication of where to expect the optimum design.

The response surface predicted by RBF interpolation using 500 data points is plotted in Figure 10. Within the design space, it was found by inspecting the modal amplitude of designs that there are three categories of transducer designs; those with the modal amplitude peak of the web mode at a frequency of less than $35 \mathrm{kHz}$, greater than $35 \mathrm{kHz}$ and at or approximately at $35 \mathrm{kHz}$. As expected, designs with the web modal amplitude peak closer to $35 \mathrm{kHz}$ are associated with high function values, thereby indicating a good performance at $35 \mathrm{kHz}$. 


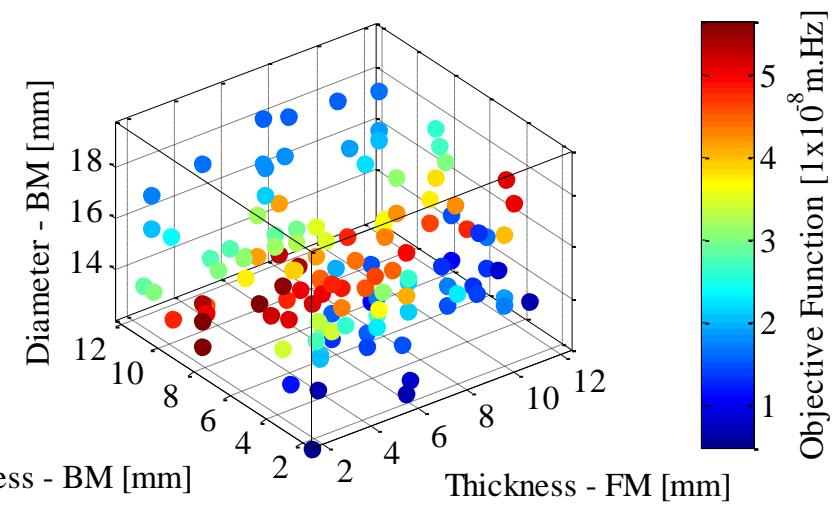

(a)

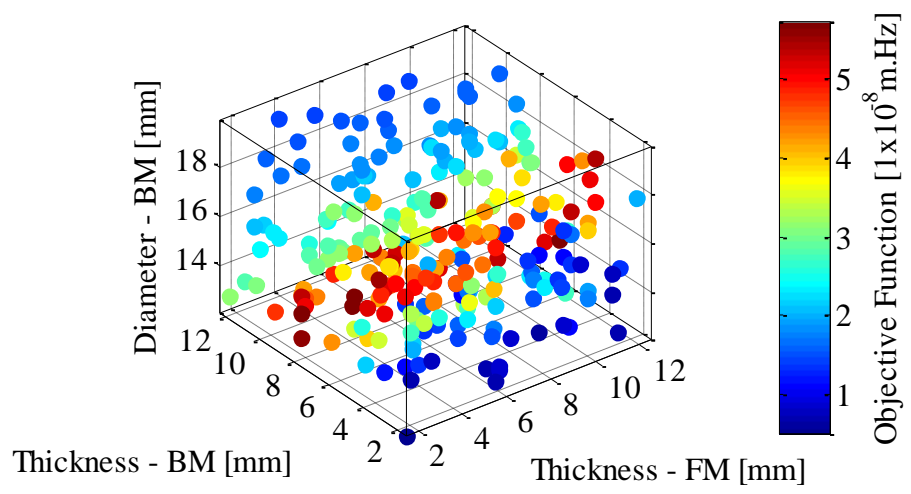

(b)

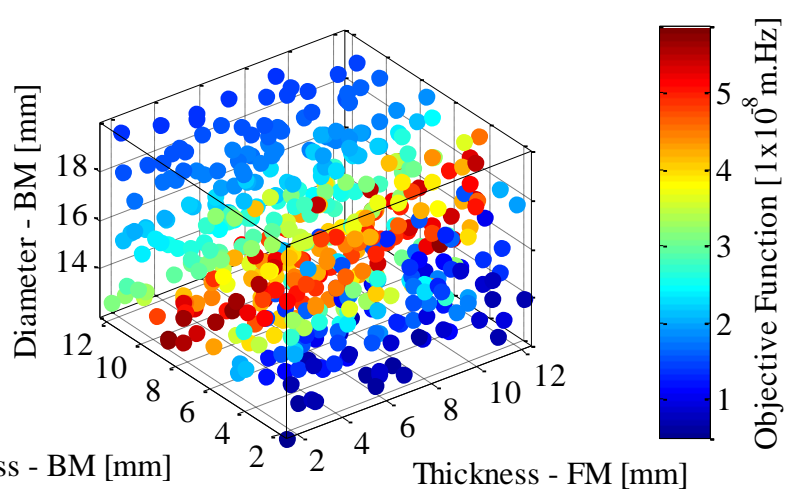

(c)

Figure 9. Latin hypercube sampled design points for (a) $\mathrm{N}=125$, (b) $\mathrm{N}=250$ and (c) $\mathrm{N}$ $=500$ points. 


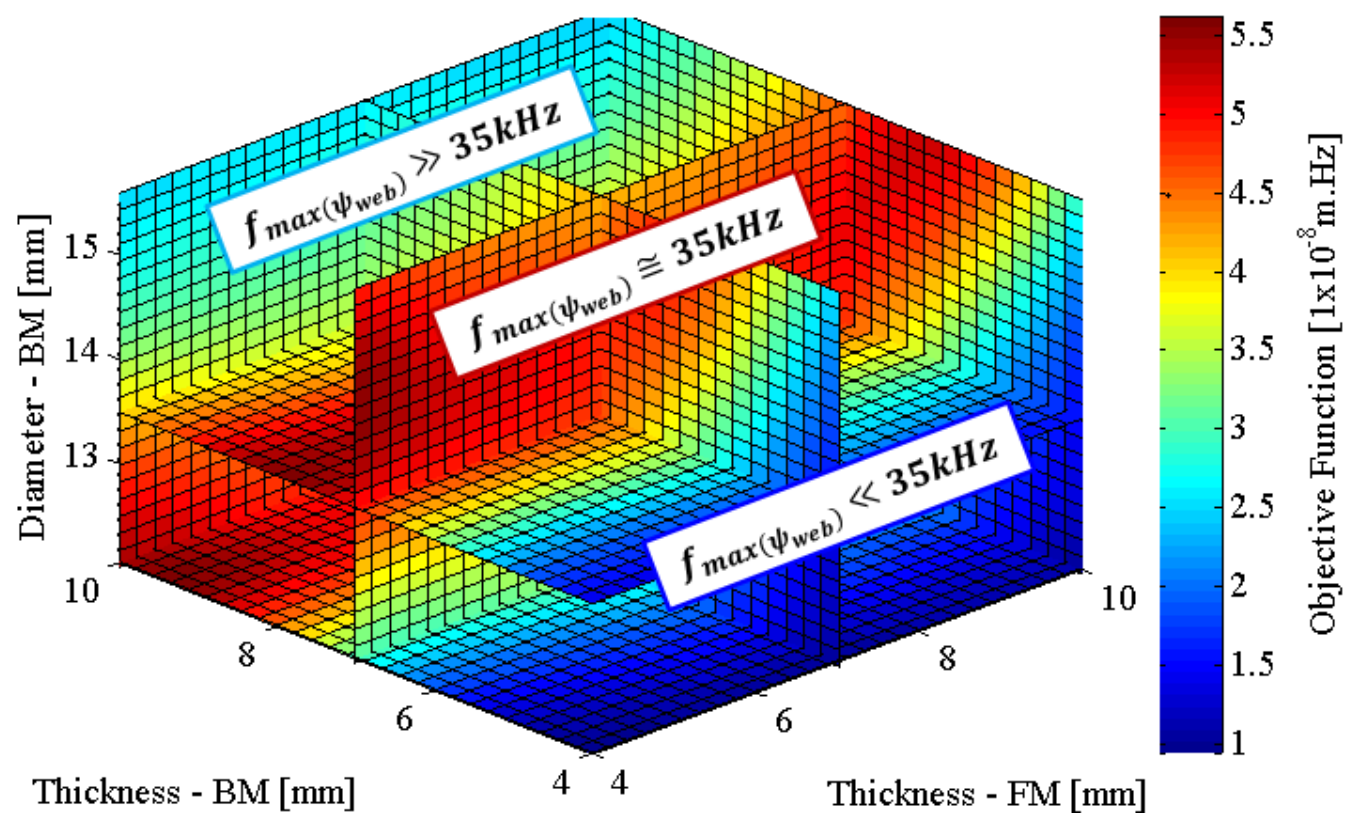

Figure 10. RBF constructed response surface that approximates the objective function.

To evaluate the accuracy of the response surface values predicted by RBF interpolation, 27 design points arranged in the form of a grid over the sampling space were selected for error analysis. The RBF predicted function values were compared to the function values computed from the Coupled SAFE-3DFE method at these 27 points. The error percentages associated with the selected points are plotted in Figures 11a-11c for the three data sets. 


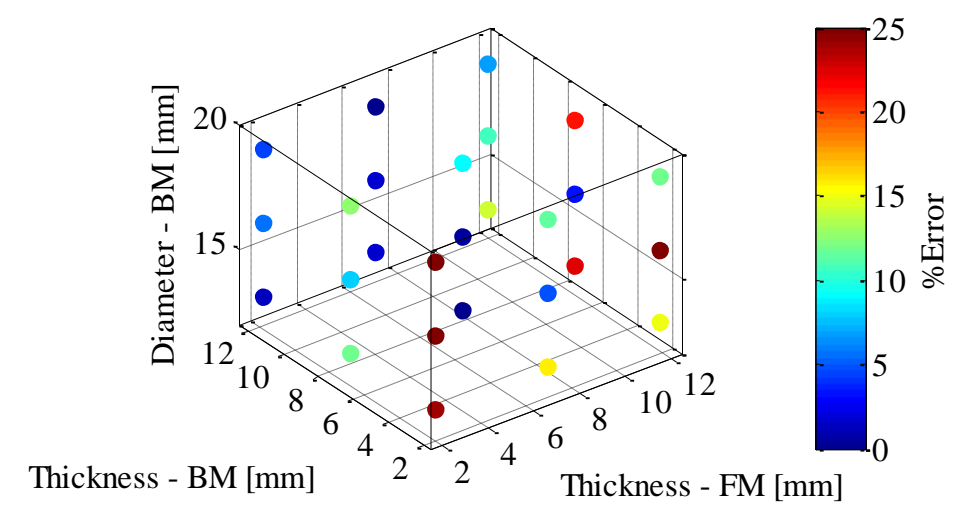

(a)

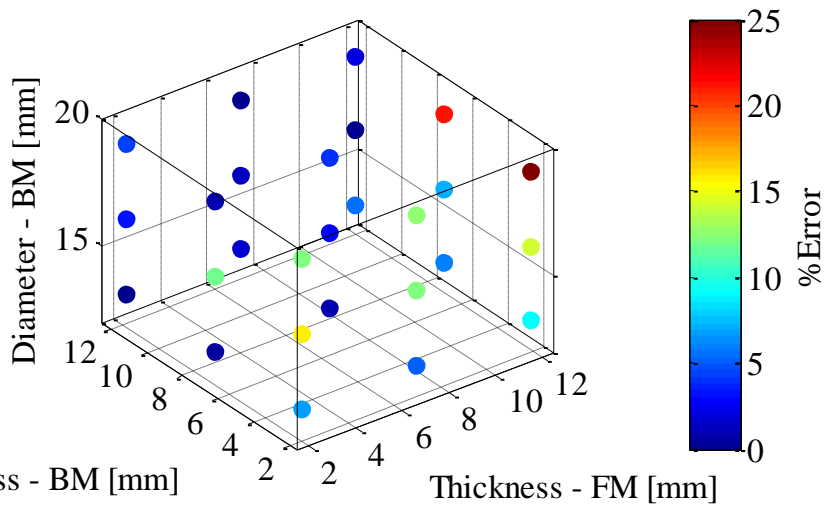

(b)

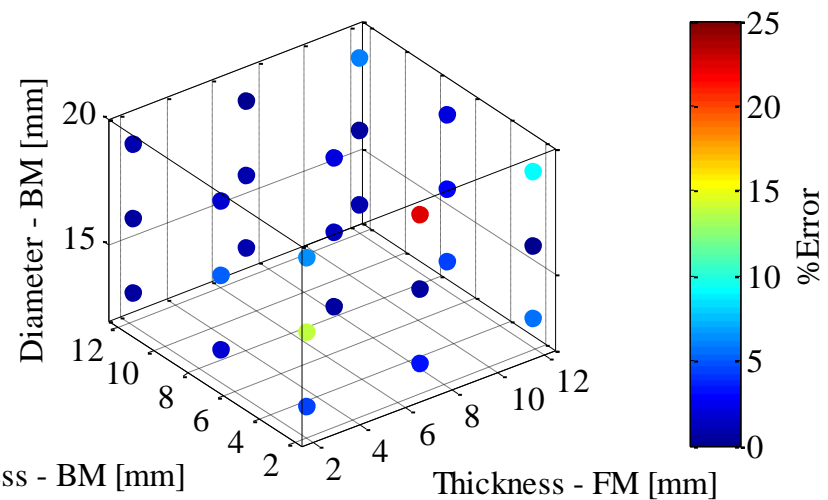

(c)

Figure 11. Errors within the sampling space associated with the predicted objective function using (a) $\mathrm{N}=125$, (b) $\mathrm{N}=250$ and (c) $\mathrm{N}=500$ sampled designs to construct the objective function.

It is clear from Figures $11 \mathrm{a}-11 \mathrm{c}$ that the errors are high at the boundary of the sampling space as compared to the interior. The errors for the three cases decrease as more sampled designs are used to approximate the objective function, thus improving the RBF predicted function values.

To demonstrate the significance of using a sampling space that is larger than the feasible design space, we evaluate the accuracy of a number of points that are within the feasible 
design space. Figures $12 \mathrm{a}-12 \mathrm{c}$ shows the errors associated with the actual design space. The errors within the design space are significantly smaller compared to those within the sampling space. Had the sampling space been the same size as the design space, large errors on the boundaries would have been inevitable as depicted in Figures 11a-11c.

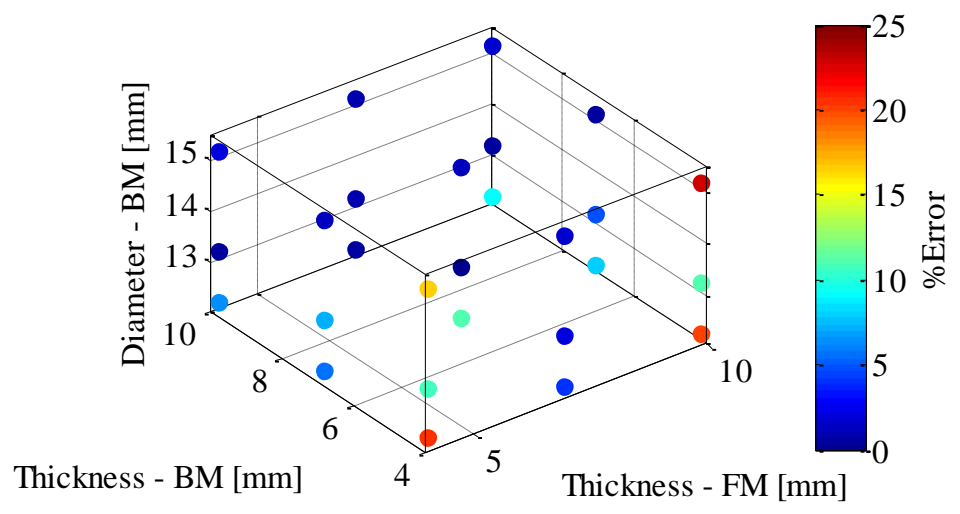

(a)

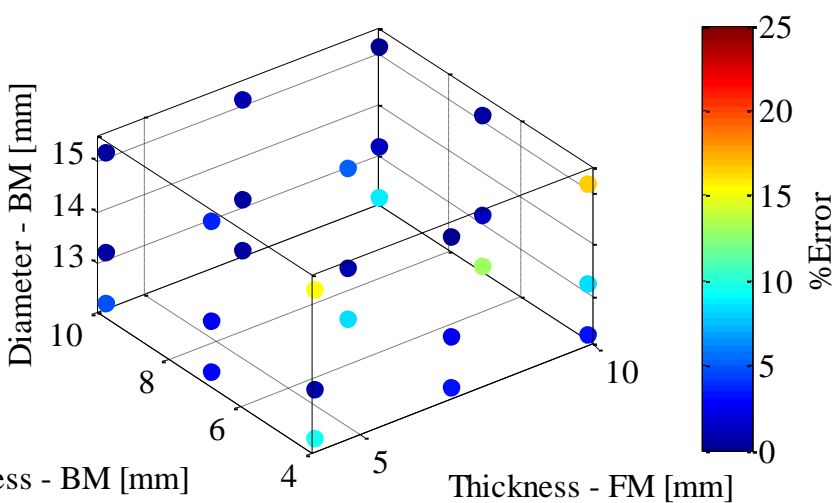

(b)

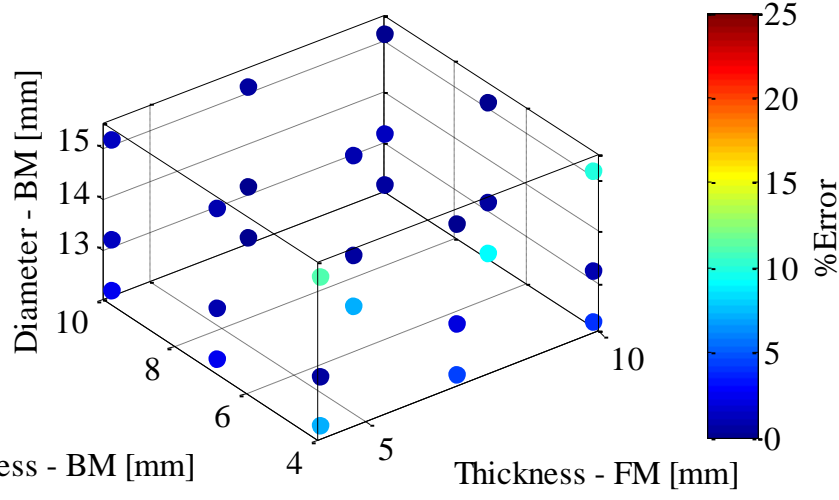

(c)

Figure 12. Errors within the design space associated with the predicted objective function using (a) $\mathrm{N}=125$, (b) $\mathrm{N}=250$ and (c) $\mathrm{N}=500$ sampled designs to construct the objective function.

The maximum error for $\mathrm{N}=500$ in the sampling and design space is $22.34 \%$ and $10.88 \%$, respectively. The larger errors in the design space could possibly be due to the evaluated 
points being very far from the sampled designs. The errors within the design space are acceptable for the purposes of this study. A response surface of the performance of different transducer designs has therefore been successfully constructed.

In optimum design, it is not always the case that the design will converge to the global maximum when more than one local maxima exist. Convergence is usually driven towards one of the local maxima, depending on where the starting point of the algorithm is situated. To capture all the local maxima in the objective function, the optimization procedure was carried out 100 times with the starting points randomly selected within the design space. Plotted in Figure 13 is a histogram of the local optimum designs predicted from the 100 runs. Each bin in the histogram represents the cost function value of a local optimum point, with the height of the bin indicating the number of times that the optimization converged to the local optimum. The three sets of data points in RBF interpolation are all considered for comparison.

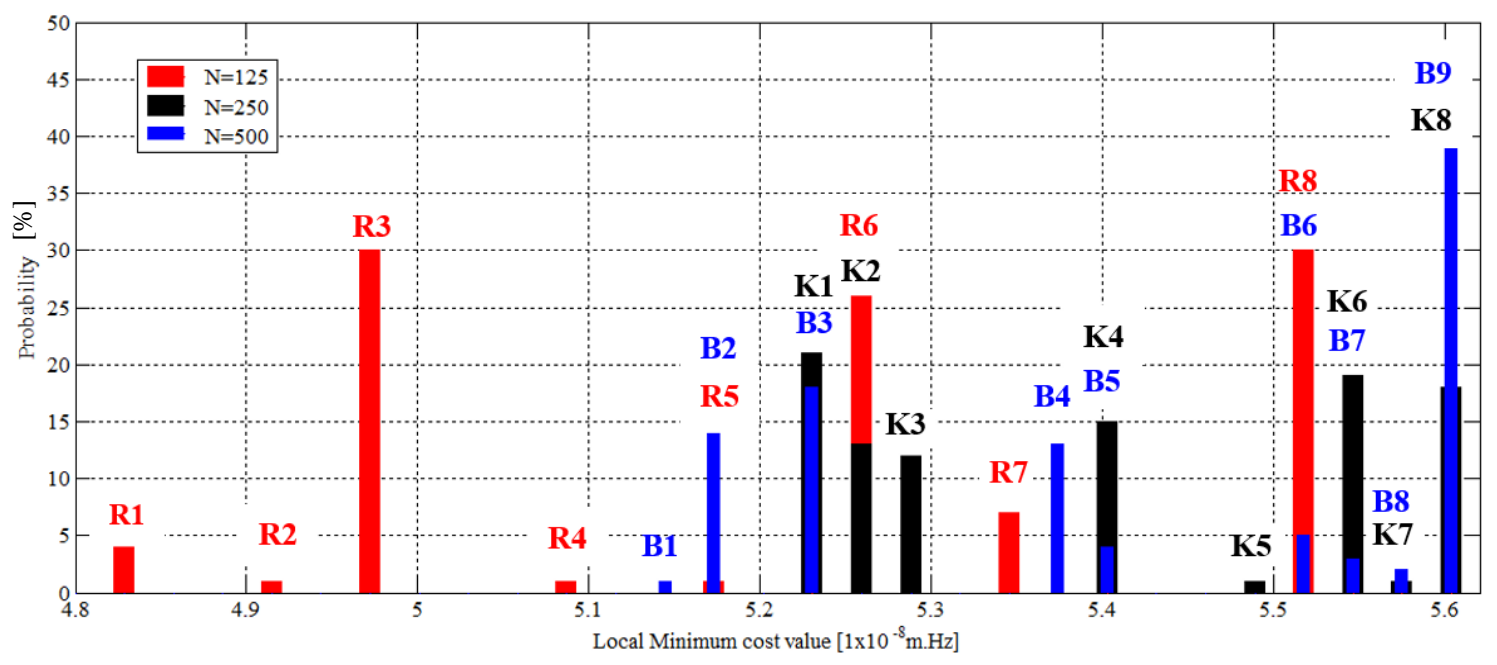

Figure 13. A histogram of the predicted local optimum designs. 

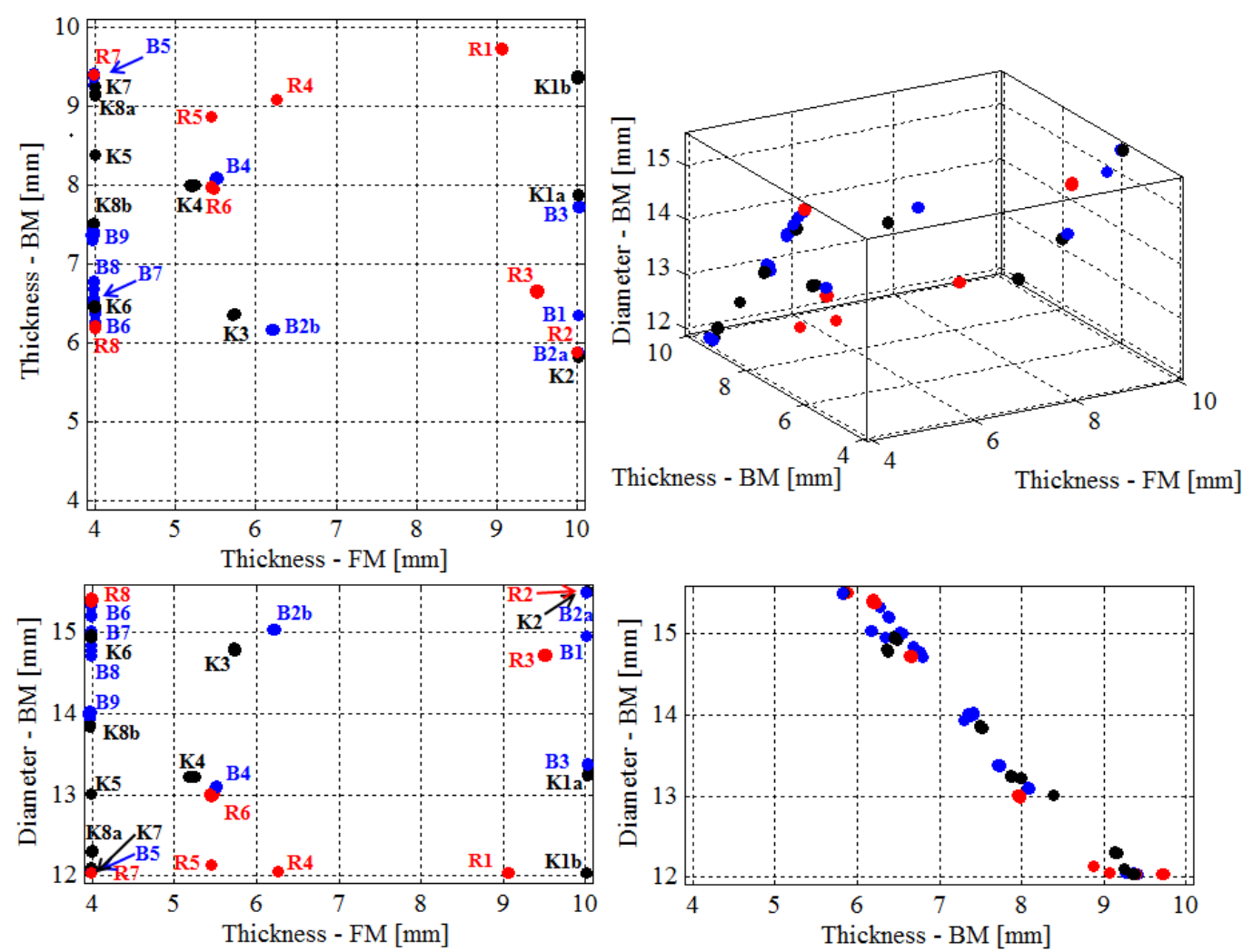

Figure 14. The location of the predicted local optima in the design space.

A single bin in the histogram may contain different types of designs, for example, bins $\mathrm{K} 1, \mathrm{~K} 8$ and $\mathrm{B} 2$ respectively contain designs $\mathrm{K} 1 \mathrm{a}$ and $\mathrm{K} 1 \mathrm{~b}, \mathrm{~K} 8 \mathrm{a}$ and $\mathrm{K} 8 \mathrm{~b}$ and $\mathrm{B} 2 \mathrm{a}$ and B2b. A similar characteristic between different designs of the same bin is the value of the objective function, implying that they have the same energy. The histogram indicates that there exist a global maximum with a function value of $5.6 \mathrm{e}-8 \mathrm{mHz}$, that was predicted in bins B9 and K8. It can be seen in Figure 14 that the designs B9 and K8b are the same, and almost on top of each other for the response surfaces constructed using $\mathrm{N}=250$ and $\mathrm{N}=500$ points.

$\mathrm{RBF}$ interpolation predicted that the energy associated with the two global optimum designs is approximately $5.6 \mathrm{e}-8 \mathrm{mHz}$. To verify the accuracy of this prediction, the 
Coupled SAFE-3DFE method was used. The results from RBF interpolation and the Coupled SAFE-3DFE method are compared in Table 1.

Table 1. Comparison of the two optimum designs.

\begin{tabular}{|l|c|c|}
\hline & Design K8a & Design K8b \\
\hline FM thickness & 4 & 4 \\
\hline BM thickness & 13.8 & \\
\hline BM diameter & & 12.3 \\
\hline RBF Objective function & 5.6114 & 5.6013 \\
\hline value & & \\
\hline Coupled SAFE-3DFE & 5.5968 & 5.5712 \\
\hline Objective function value & & 0.5403 \\
\hline |\%Error| & & \\
\hline
\end{tabular}

It is evident from the table that the response surface represented by the RBFs is accurate at the optimal design points. The finite element models of the two optimum designs are plotted in Figures 15a and 15b, respectively.

Table 1 shows that the optimum designs lie on the boundary of the design space, where the front mass thickness is $4 \mathrm{~mm}$. This is due to the performance of the transducer being greatly influenced by a decrease in the front mass thickness. Therefore, a better design would have been obtained if the front mass was not constrained to a lower bound of $4 \mathrm{~mm}$. A number of designs were perturbed around K8a design by decreasing and increasing the 
three design variables respectively. It was found that a $5 \%$ decrease in the front mass thickness leads to a $1.56 \%$ increase in the objective function value. An increase in the front mass thickness as well as changes to the back mass size results in a decrease in the objective function value. This confirms that the design is at least a local maximum.

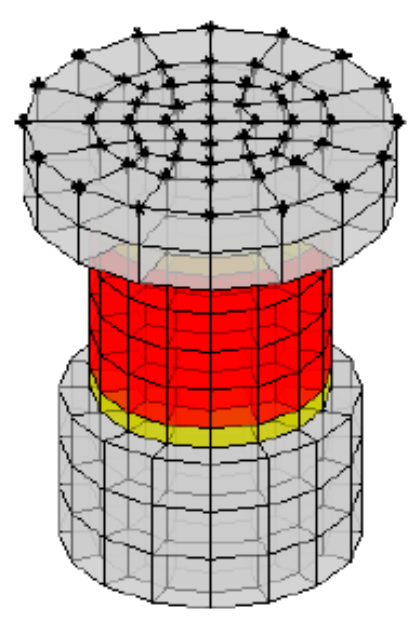

(a) Design K8a

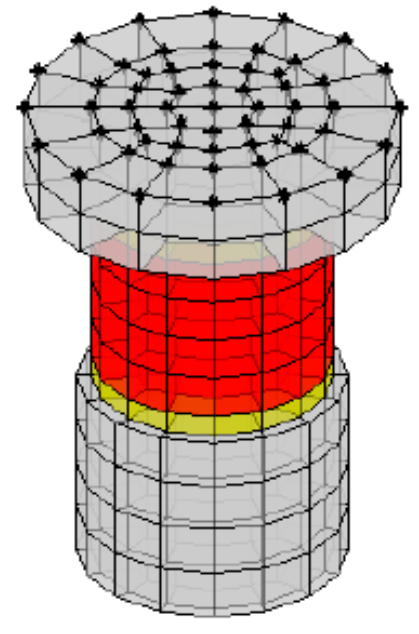

(b) Design K8b

Figure 15. The finite element models of the two distinct optimum designs that have the same optimum objective function value.

\subsection{Guided Wave Analysis of the Optimal Design}

To evaluate the accuracy of the Coupled SAFE-3DFE method when applied to K8a design, experimental measurements were carried out in the lab. The K8a transducer was bonded to the web of an available $5 \mathrm{~m}$ long UIC60 rail segment, located at a distance of $1.5 \mathrm{~m}$ from one end. The experimental measurement was carried out using a 17.5 cycle hanning-windowed tone-burst at $35 \mathrm{kHz}$ excitation. The unit amplitude voltage signals across each of the 4 piezoelectric rings were amplified to a peak amplitude of 20Volts. The horizontal displacement response at the centre of the rail web was measured at a distance of $1.5 m$ from the transducer location. A Polytec PSV- 400 laser vibrometer was 
used to perform this experimental measurement. The output displacement was then scaled by a factor of $1 / 80$ to compare with modelling results from Coupled SAFE-3DFE, where a $1 V$ peak amplitude excitation signal, across the four piezoelectric rings, was used.

The experimental results are compared to Coupled SAFE-3DFE results in Figure 16.

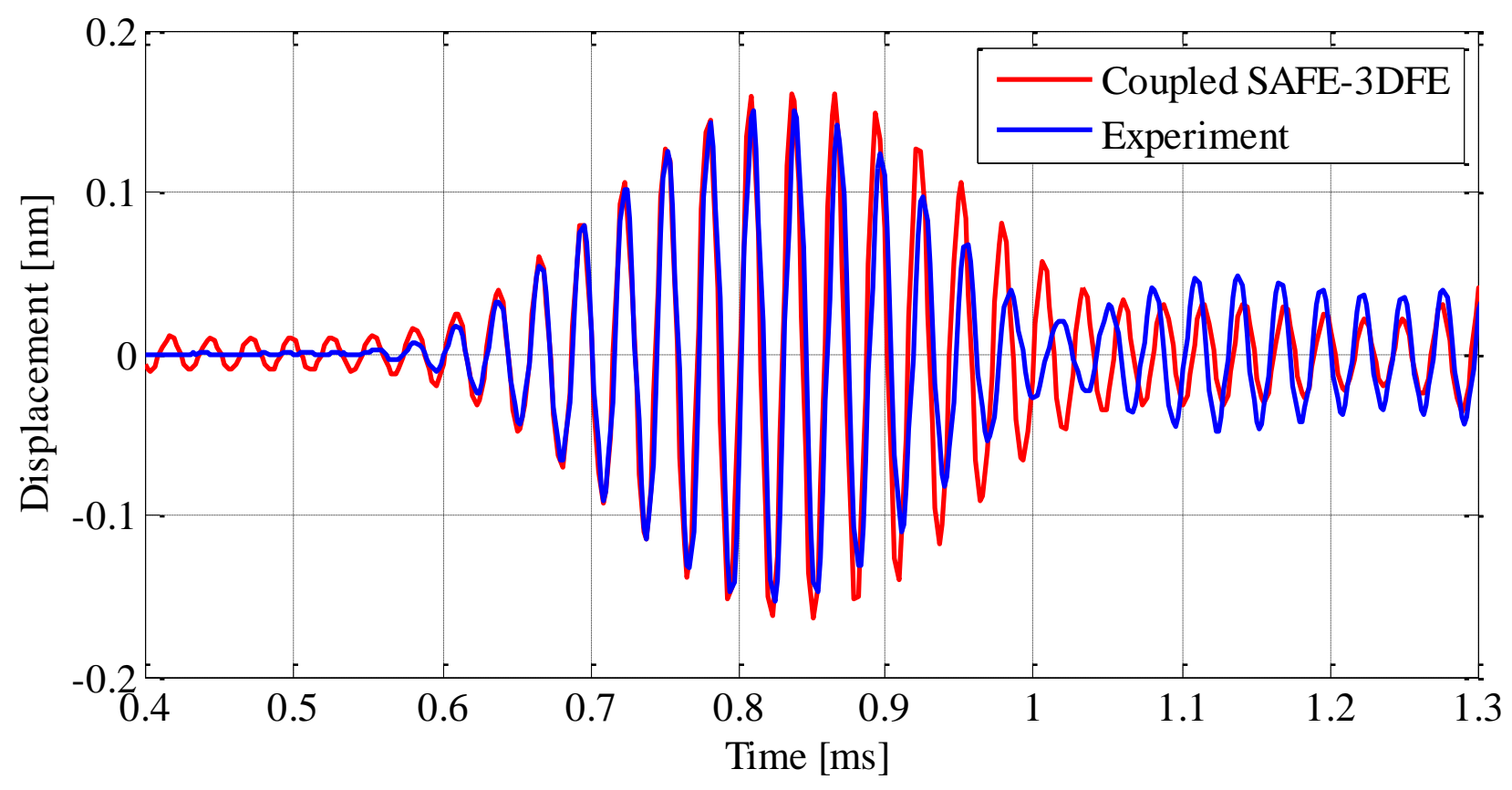

Figure 16. Comparison of Coupled SAFE-3DFE results to experimental results.

The results demonstrate an exceptional agreement between the Coupled SAFE-3DFE prediction and the experimental measurements.

The manufactured transducer was also tested on an operational rail line, Figure 17a. A pulse-echo measurement was performed using a 17.5 cycle tone burst with a $35 \mathrm{kHz}$ centre frequency as the excitation signal. The short time Fourier transform was used to produce the spectrogram shown in Figure 17b. The spectrogram clearly shows reflections from aluminothermic welds in the rail at different distances from the transducer. There are additional reflections which correspond to multiple reflections and these are not 
labelled in the figure. For example, the reflection arriving slightly before $0.3 \mathrm{~s}$ (reflection D), is produced by a weld at a distance of $420 \mathrm{~m}$ from the transducer. The variation of arrival time with frequency corresponds to the group velocity of the web mode, computed by the SAFE method, thereby confirming that the transducer effectively excites and senses this mode of propagation.

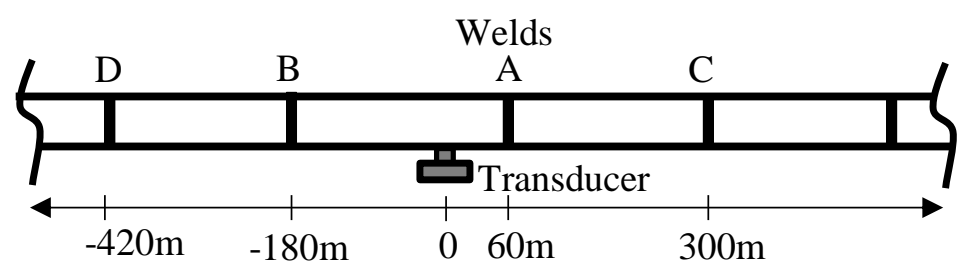

(a)

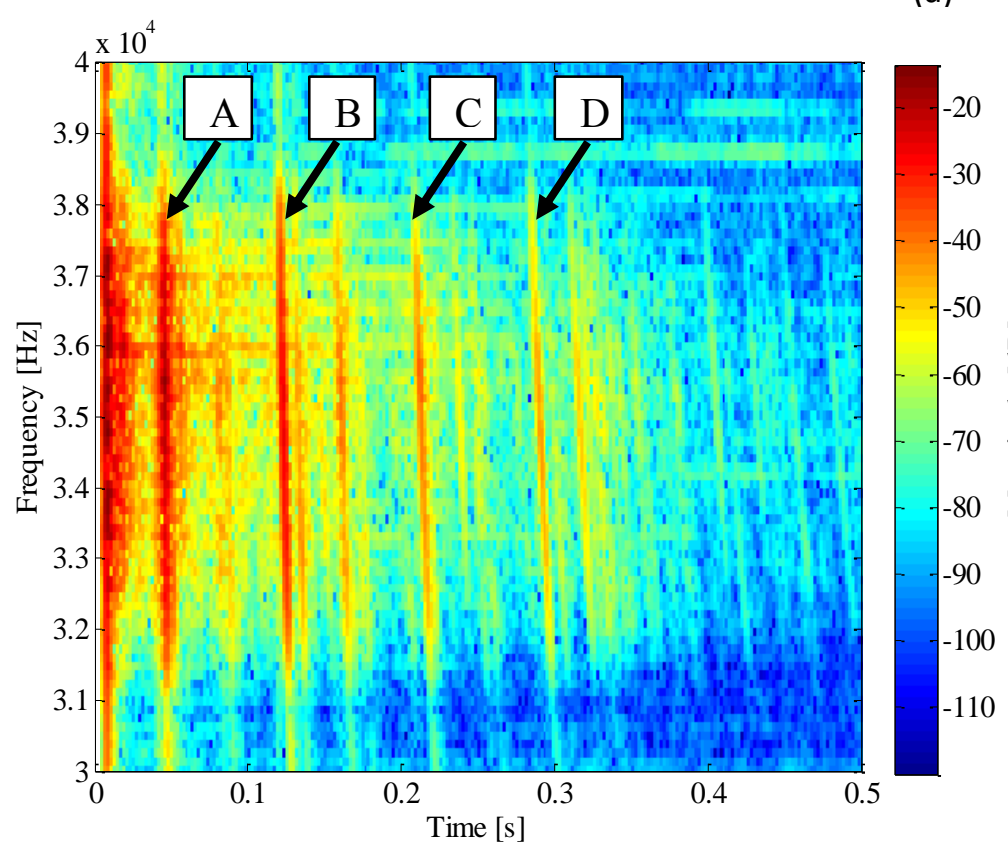

(b)

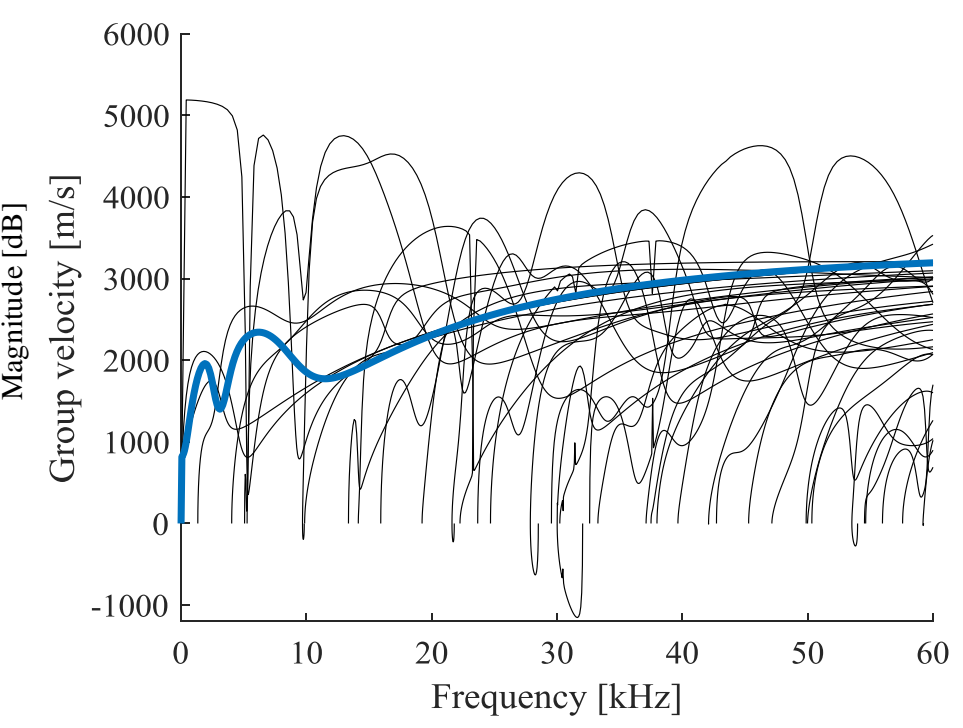

(c)

Figure 17. (a) A schematic representation of the operational railway line, (b) a spectrogram of the web mode reflections from aluminothermic welds at A:60m, B:180m, C:300m and D:420m, when the rail web is excited by the K8a transducer and (c) the group velocity of the rail with the web mode highlighted. 


\section{Conclusion}

In this study, a computationally efficient design optimization procedure for optimal transducer design was developed, demonstrated and validated. The computationally efficient procedure relied on the computational benefits of Coupled SAFE-3DFE as well as the ability to conduct multiple analyses concurrently that response surface methodologies enabled. The importance of ensuring that the sampling domain for the response surface is larger than the feasible design domain was highlighted. This ensured an accurate response surface close to edges of the feasible design domain. The performance of the optimal transducer design had exceptional agreement with the measured performance of the manufactured transducer.

\section{References}

[1] Wilcox P, Pavlakovic B, Evans M, Vine K, Cawley P, Lowe M, et al. Long Range Inspection of Rail Using Guided Waves. In: Thompson DO, Chimenti DE, editors. Rev. Prog. Quant. Nondestruct. Eval., vol. 22, American Institute of Physics; 2003, p. 236-43.

[2] Loveday PW, Long CS. Long Range Guided Wave Defect Monitoring in Rail Track. In: Thompson DO, Chimenti DE, editors. Rev. Prog. Quant. Nondestruct. Eval., vol. 1581, American Institute of Physics; 2014, p. 179-85.

[3] Long CS, Loveday PW. Prediction of Guided Wave Scattering by Defects in Rails Using Numerical Modelling. AIP Conf Proc 2014;1581:240-7.

[4] Loveday PW. Analysis of Piezoelectric Ultrasonic Transducers Attached to Waveguides Using Waveguide Finite Elements. IEEE Trans Ultrason Ferroelectr Freq Control 2007;54:2045-51.

[5] Loveday PW. Simulation of piezoelectric excitation of guided waves using 
waveguide finite elements. IEEE Trans Ultrason Ferroelectr Freq Control 2008;55:2038-45.

[6] Hayashi T, Song W-J, Rose JL. Guided wave dispersion curves for a bar with an arbitrary cross-section, a rod and rail example. Ultrasonics 2003;41:175-83.

[7] Gavric L. Computation of Propagative Waves in Free Rail Using a Finite Element Technique. J Sound Vib 1995;185:531-43.

[8] Ramatlo DA, Long CS, Loveday PW, Wilke DN. SAFE-3D analysis of a piezoelectric transducer to excite guided waves in a rail web. In: Chimenti DE, Bond LB, editors. Rev. Prog. Quant. Nondestruct. Eval., American Institute of Physics; 2016, p. 020005-1 - 020005-10.

[9] Arora JS. Introduction to Optimum Design. 2nd ed. London: Elsevier Academic Press; 2012.

[10] Allik H, Hughes J. Finite Element Method for Piezoelectric Vibration. Int J Numer Methods Eng 1970;2:151-7.

[11] Tang B. Orthogonal array-based Latin hypercubes. J Am Stat Assoc $1993 ; 88: 1392$.

[12] Helton J, Davis F, Johnson J. A Comparison of Uncertainty and Sensitivity Analysis Results Obtained with Random and Latin Hypercube Sampling. Reliab Eng Syst Saf 2005;89:305-30.

[13] McDonald D, Grantham W, Tabor W, Murphy M. Global and Local Optimization using Radial Basis Function Response Surface Models. Appl Math Model 2007;31:2095-110.

[14] Rippa S. An algorithm for selecting a good value for the parameter $\mathrm{c}$ in radial basis function interpolation. Adv Comput Math 1999;11:193-210. 
[15] Wright MH. Nelder, Mead, and the Other Simplex Method. Doc Math 2012;ISMP:271-6. 